needed two revisions of boot size. The gangrenous tips were being extruded by the growth of new toe (Fig. 3).

\section{Discussion}

We present this case because of the difficulties we experienced planning rational investigation and treatment. In the absence of autoimmune disease, peripheral vascular disease in such a young child is rare. ${ }^{1}$ In the presence of rapidly advancing gangrene, comprehensive investigation before treatment was impossible and many of the studies, particularly those of fibrinolysis, were carried out retrospectively.

One of the boy's relatives who had died developed ischaemia in her early teens, and the disease pursued a relentless course. Investigations included most of the above, together with skin biopsies and histological examination of amputated digits. These had failed to indicate a diagnosis. She had been treated with steroid and immunosuppresive drugs, to no avail. The raised fibrinogen, erythrocyte sedimentation rate, and high platelet count in our patient, however, suggested an autoimmune process, despite the absence of circulating immune complexes. He was therefore offered steroid treatment; again with no effect.

In the six years since the relative died, new techniques for the investigation of thrombosis and firbrinolysis have become available in certain centres in the United Kingdom (protein $\mathrm{C}$ activity was measured in Amsterdam). The good peripheral pulses in our patient directed our attention to these factors which often affect the microvascular circulation. Antithrombin III and protein $\mathrm{C}$ activity are known to be involved in familial disorders, but in both these deficiency states the thrombotic process usually affects bigger vessels, and we were not surprised that concentrations proved normal. Our investigations could not be carried out before treatment. We hope that this experience will enable us to work early with an established protocol towards a diagnosis should this child relapse.

Prostacyclin is a powerful vasodilator and the most potent known natural inhibitor of platelet aggregation in man. Biological and pharmacological effects were reviewed recently. ${ }^{2}$ There has been considerable experience in the use of Prostacyclin for peripheral vascular disease in adults ${ }^{3}$ but its use for this purpose in children has not been reported.

We are convinced that the drug saved the feet of this child, and indeed that a less cautious dose increment could possibly have effected more recovery. An infusion rate of $12 \mathrm{ng} / \mathrm{kg}$ per minute for several hours caused no side effects. We are similarly greatly impressed by the continued healing and regrowth of the toes several weeks after stopping treatment which seems analogous to the growth of children's digits after partial amputation. ${ }^{4}$

We thank Professor C Prentice and Dr A Davies of Leeds General Infirmary for their help with fibrinolysis studies and Dr J Belch of Glasgow Royal Infirmary for advice.

\footnotetext{
References

1 Turpie AGG, Forbes CD, McNicol GP. Idiopathic gangrene in African children. Br Med J 1967:iii:646-8.

2 Prostacyclin, thromboxane and leukotreines. $\mathrm{Br}$ Med Bull 1983;3:39.

3 Belch JJF, Drury JK, Copell H, et al. Intermittent epoprostenol (Prostacyclin) infusion in patients with Raynaud's syndrome. Lancet 1983:i:313-6.

4 Illingworth CM. Trapped fingers and amputated finger tips in children. J Pediatr Surg 1974;9:853-9.
}

Correspondence to Dr C de San Lazaro, Department of Child Health, Royal Victoria Infirmary, Newcastle upon Tyne NE1 4LP.

Received 14 November 1984

\title{
Hyperbaric oxygenation in peripheral ischaemic lesions in infants
}

\author{
E ROSENTHAL, A BENDERLY, I MONIES-CHASS, J FISHMAN, J LEVY, AND V BIALIK
}

Department of Paediatrics A, Paediatric-Orthopaedic Unit, and Department of Anaesthetics, Rambam Medical Center and Faculty of Medicine, Technion-Israel Institute of Technology, Haifa, Israel

SUMmaRY Acute peripheral arterial occlusion may lead to gangrene, with loss of parts of arms and legs. Three infants with disseminated intravascular clotting developed dark red discoloration of the tips of fingers and toes which progressed proximally. Repeated hyperbaric oxygenation treatments caused regression of the demarcation line and further progression of necrosis stopped.

Acute massive peripheral arterial occlusion secondary to disseminated intravascular clotting is a rare and severe complication in infants which may lead to gangrene and loss of arms and legs, or death. In the past 10 years we treated three infants with this disorder and peripheral ischaemia with hyperbaric oxygenation in an effort to prevent and reverse hypoxic changes. All three patients shared a common clinical course and we report the first patient here. 


\section{Case report}

This 7 month old girl was admitted to hospital for severe dehydration due to diarrhoea. Normal values were obtained for serum electrolytes, urea, haemoglobin, white blood count, prothrombin time, partial thromboplastin time, platelets, and creatinine. Stool cultures grew pathogenic Escherichia coli.

Treatment with fluids, electrolytes, and antibiotics did not improve her clinical condition. After two weeks, generalised purpura was noticed and laboratory studies showed: haemoglobin $3.6 \mathrm{mmol} / \mathrm{l}$; platelet count $14 \times 10^{9} / 1$, prothrombin time 0.34 , partial thromboplastin time $39^{\prime \prime}$. Fibrinogen split products were raised, although fibrinogen was $2 \cdot 77$ $\mathrm{g} / \mathrm{l}$. Treatment with fresh frozen plasma and packed cells did not halt the disease process.

Subsequently, we noticed a dark red discolouration of all fingers and toes, and several tips in all four limbs gradually turned necrotic. Discolouration continued to extend proximally. In the hands the demarcation line reached the mid and distal phalanges. In the legs it reached the mid-tibial area (Fig. 1).

Angiography on the legs did not show any anomaly of the popliteal arteries and the trifurcation on both sides. There was, however, a progressive narrowing of the distal arteries.

It seemed that loss of extended parts of arms and legs was inevitable. The infant was drenched in a hyperbaric chamber at a pressure of 2.5 atmospheres of pure oxygen twice daily (two hours each session). We observed a rapid improvement in skin colour in all affected areas (except the dark necrotic ones) within a few minutes after beginning treatment. This improvement persisted for a short time after decompression, but turned dark again during the next few hours.

The demarcation line, however, between normal and abnormal skin gradually receded distally after each treatment, while progression of dark necrotic areas stopped completely. After 10 days of treatment it seemed that maximal benefit had already been achieved since the demarcation line reached the dark necrotic areas and was clear cut (Fig. 2). At that time the patient developed an episode of generalised seizures attributed to oxygen poisoning and we decided to stop the treatment at once.

The infant underwent surgery to remove the necrotic tissue and partial amputation of feet was performed. The child is currently standing without support and uses orthopaedic shoes without prosthesis.

The other two patients had a similar clinical course. One 18 and the other 24 months old, had peripheral ischaemia of one lower limb and extended

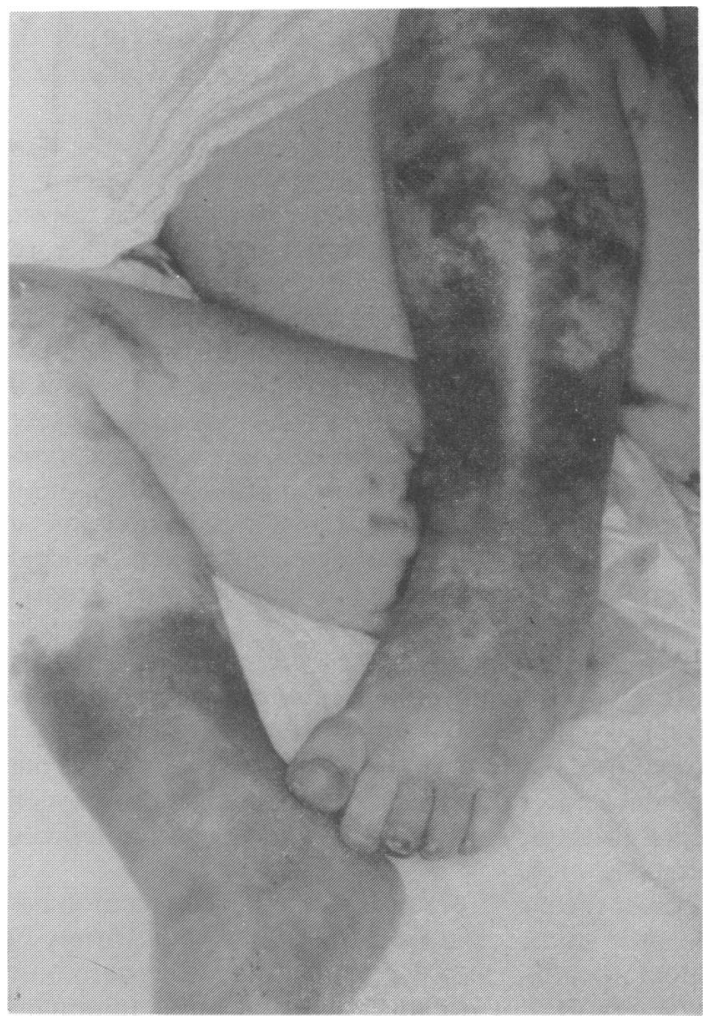

Fig. 1 Ischaemic lesions in the legs and feet before hyperbaric oxygenation treatment.

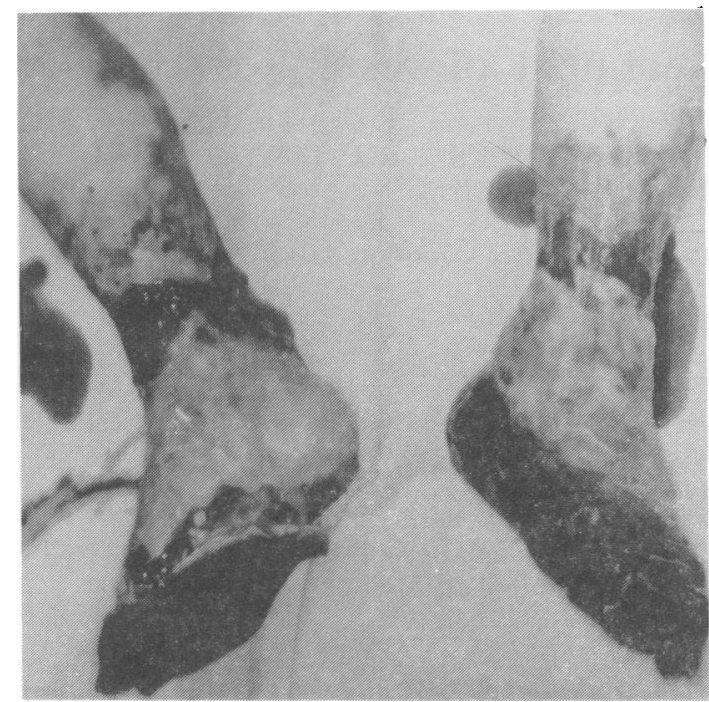

Fig. 2 Appearance of the legs and feet after treatment. Note the demarcation line between the normal and the necrotic areas. 
areas of necrosis below the knee. As a result of hyperbaric oxygenation the limbs were saved ending with a partial foot amputation only.

\section{Discussion}

The patients reported here presented with the rare and most severe variant of disseminated intravascular clotting: sudden occlusion of multiple peripheral arterioles by massive microthrombosis. This phenomenon has been variously described as purpura fulminans or purpura gangrenosa ${ }^{1}$ and has its onset after various infections. Massive areas of haemorrhage in the skin, usually of the arms and legs, may progress to extensive gangrene. There is, however, a wide marginal zone of hypoxia due to partial arteriolar occlusion which may benefit by hyperbaric oxygenation treatment.

The extensive use of this treatment in humans is restricted by the development of bronchopulmonary dysplasia and convulsions. ${ }^{2}$ The development of oxygen toxicity may be delayed by interposing regular, intermittent periods of normal oxygen levels without concurrently reducing the beneficial effects. $^{3}$

Over the past 20 years hyperbaric oxygenation has become an important treatment in a wide variety of anoxic conditions. ${ }^{4}$ We could find, however, only two reports of infants with purpura fulminans treated with this. ${ }^{5}{ }^{6}$ Both had a much milder course with no loss of limbs. In those cases the authors felt that spontaneous recovery might have occurred anyway, and the treatment mainly served to shorten the duration of the disease.

In the patients presented here, however, we believe that hyperbaric oxygenation had a major influence in reducing the areas doomed for amputation. The good clinical results suggest that hyperbaric oxygenation may have a major treatment application in this serious condition.

\footnotetext{
References

1 Urbaniak JR, O'Neil MT, Meyer LC. Purpura fulminans. J Bone Joint Surg 1973;55:69-77.

2 Lambertson CJ. Effects of oxygen at high partial pressure. In: Haffner FG, ed. Handbook of physiology of respiration, vol 2. Baltimore: Williams and Wilkins, 1965:1027.

${ }^{3}$ Miller JH, Mendoza SA. Protection against the toxic effects of hyperbaric oxygen by intermittent exposure to reduced oxygen pressures. Pediatr Res 1978;12:283-7.

${ }^{4}$ Gunby P. Evaluation continues of $\mathrm{HBO}$ clinical uses. JAMA 1981;246:1059-64.

5 Waddell WB, Saltzman HA, Fuson RL, Harrison J. Purpura gangrenosa treated with hyperbaric oxygenation. JAMA 1965; 191:971-4.

6 Kuzemko JA, Loder RE. Purpura fulminans treated with hyperbaric oxygen. Br Med J 1970;iv:157.
}

Correspondence to Dr A Benderly, Department of Paediatrics A, Rambam Medical Center, Haifa 35254, Israel.

Received 3 January 1985

\title{
Treatment of respiratory papillomatosis with adenine arabinoside
}

\author{
W A HENDRICKSE, B C IRWIN, R J LEVINSKY, C M BAILEY, G TYMM, \\ AND J N G EVANS
}

Hospital for Sick Children, Great Ormond Street, and Institute of Child Health, London

SUMMARY Nine children with respiratory papillomatosis have been treated with adenine arabinoside with limited success. No long term benefit was obtained in six, and although resolution was achieved in one and probably two others, this could not be attributed confidently to treatment.

Respiratory papillomatosis (or laryngeal papillomatosis) are histologically benign tumours that usually arise in the larynx but may spread throughout the respiratory tract. Consequently, the disease may be life threatening if the airway is impaired. The mainstay of management is surgical but if the disease is widespread complete removal may be impossible. A viral aetiology has long been suspected and recently evidence has been obtained of an association between respiratory papillomata and deoxyribonucleic acid (DNA) containing human papilloma virus. ${ }^{12}$ Treatment with an anti-DNA virus agent, therefore, seems rational. ${ }^{3}$ Adenine arabinoside is an established anti-DNA viral agent but there have been no published reports giving detailed results of its use in treating this condition.

We therefore reviewed the case records of all 